\title{
The extended surface forces apparatus. IV. Precision static pressure control
}

\author{
E. Schurtenberger and M. Heuberger ${ }^{\mathrm{a})}$ \\ Empa, Materials Science and Technology, CH-9014 St. Gallen, Switzerland
}

(Received 24 June 2011; accepted 15 September 2011; published online 14 October 2011)

\begin{abstract}
We report on design and performance of an extended surface forces apparatus (eSFA) built into a pressurized system. The aim of this instrument is to provide control over static pressure and temperature to facilitate direct surface force experiments in equilibrium with fluids at different loci of their phase diagram. We built an autoclave that can bear a miniature eSFA. To avoid mechanical or electrical feedtroughs the miniature apparatus uses an external surface coarse approach stage under ambient conditions. The surface separation is thus pre-adjusted to approximately $\sim 3 \mu \mathrm{m}$ before sliding the apparatus into the autoclave. Inside the autoclave, the surface separation can be further controlled with a magnetic drive at sub-Ångstrom precision over a $14 \mu \mathrm{m}$ range. The autoclave pressure can then be set and maintained between 20 mbar and 170 bars with few mbar precision. The autoclave is connected to a specially designed pressurization system to precondition the fluids. The temperature can be controlled between -20 and $60{ }^{\circ} \mathrm{C}$ with few $\mathrm{mK}$ precision. We demonstrate the operation of the instrument in the case of gaseous or liquid carbon dioxide. Thanks to a consequent decoupling of the eSFA mechanical loop from the autoclave structure, the obtained measurement stability and reproducibility, at elevated pressures, is comparable to the one established for the conventional eSFA, operated under ambient conditions. (C) 2011 American Institute of Physics. [doi:10.1063/1.3648121]
\end{abstract}

\section{INTRODUCTION}

Interactions between molecules and surfaces play a chief role in many fields of science such as colloid science, biology, geological processes, tribology as well as technical applications and processes. There are several techniques to directly and quantitatively measure surface forces across a medium. Besides the surface forces apparatus (SFA), ${ }^{1}$ examples include the thin film balance, ${ }^{2}$ the micro-electromechanical system force sensor, ${ }^{3}$ the atomic force microscope (e.g., evanescent wave AFM) ${ }^{4}$ the particle interaction apparatus, ${ }^{5}$ and the piezoresistive cantilevers. ${ }^{6}$ In each of the mentioned techniques a deflection of a compliant part is measured to determine the force. These techniques are usually used under ambient condition, which implies a serious limitation in terms of thermodynamic loci. Namely, has it not yet been possible to measure and study direct surface forces in technically relevant high-pressure fluids like carbon dioxide, supercritical water, fluid methane, or lubrication oils.

One pioneering instrument is described in literature that provides sliding friction measurements in pressurized carbon dioxide to study refrigerant lubrication. It can be operated at pressures up to 140 bars and in a temperature range between 0 and $100{ }^{\circ} \mathrm{C} .7,8$

Over the past 20 years, there have been some notable advances to extend some of the above mentioned direct-force techniques to include vacuum conditions-mainly for cleanliness, or, provide a range of low temperatures.

The Binnig group, for example, built the first lowtemperature AFM under vacuum conditions. ${ }^{9}$ This AFM was operated at $4.2 \mathrm{~K}$ and $3 \times 10^{-11}$ mbar. Another thermostatic

a)Electronic mail: manfred.heuberger@empa.ch.
AFM was realized by Pang et al. ${ }^{10}$ There, the sample temperature can be varied in a range between -90 and $20{ }^{\circ} \mathrm{C}$ with $\pm 1{ }^{\circ} \mathrm{C}$ precision and the pressure is lowered to 1.3 $\times 10^{-10}$ mbar.

We have found no literature that a direct surface force measurement has yet been realized at controlled pressures above 1 bar. In this paper, we present a new type of an extended surface forces apparatus (eSFA) where both the temperature as well as the pressure can be controlled. Our experimental window is defined by the following values: -20 to $60{ }^{\circ} \mathrm{C}$ with a stability of better than $0.002{ }^{\circ} \mathrm{C} / \mathrm{h}$ and pressure $0.02-170$ bars with a stability of $2 \mathrm{mbar} / \mathrm{h}$. Within these thermodynamic parameters, we demonstrate measurement of surface forces in equilibrium with the bulk fluid at different loci of its phase diagram.

\section{INSTRUMENTAL DESIGN}

In this paragraph, we first give a more general overview of the most important components and their function. In a second part, we shall then describe selected novel elements of the instrument in more detail.

In analogy to the conventional SFA, ${ }^{1}$ the surface force is to be measured between two atomically smooth surfaces of mica. The 3-5 $\mu \mathrm{m}$ thick mica surfaces are approached one another in crossed cylinder configuration. One of the surfaces is mounted on a spring, which is the force-sensitive element in the mechanical loop (Fig. 1(a)). The distance between the surfaces is measured using white light interferometry. To raise the optical finesse of the thin film interferometer, formed by the two mica layers and the medium between them, the micas are provided a thin silver layer on the outer faces, each. To simplify the optics setup we chose to use the light reflected 
back from the interferometer. This is in contrast to many conventional SFA designs where the transmitted light is analyzed.

To simplify sealing, we also decided to omit mechanical or electrical feedthroughs, apart from one single optical window.

The measurement of surface forces with an SFA is based on controlling the surface separation between the surfaces over several micrometer ranges with sub-nanometer precision. We have designed a magnetic drive with minimal thermal dissipation for stability. Since the magnetic drive has a limited $14 \mu \mathrm{m}$ range it is required to pre-position the surfaces at a separation of 3-5 $\mu \mathrm{m}$ prior to insertion into the autoclave. This pre-positioning is done with the pre-positioning stage illustrated in Fig. 1(b). It consists of a separate mechanical loop, which securely holds the two elements of the miniature eSFA for positioning.

Later, the autoclave is connected to a larger pressurization vessel via steel piping. The pressurization vessel is used to precondition the medium to the desired pressure at no loss of purity. It consists of a steel container with an inner steel bellow that allows pressurizing the medium of choice with nitrogen from the outside, but without mixing the media. The bellow - for example filled with carbon dioxide-is compressed with gaseous nitrogen. We are using a computer controlled reducing valve. For convenience, the pressurization vessel and the autoclave are located inside separate temperature controlled thermal insulation boxes. (a)

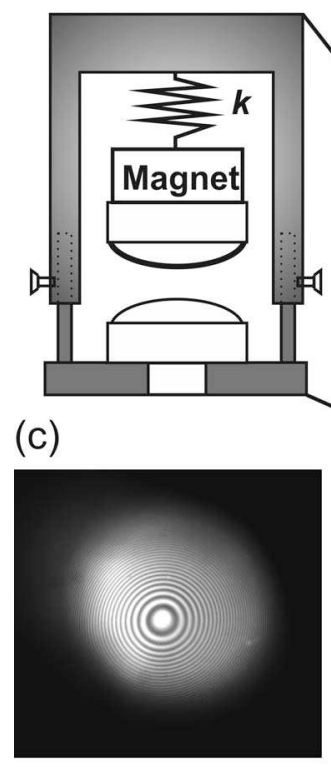

(b)

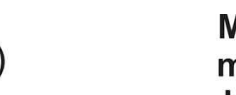

meter head
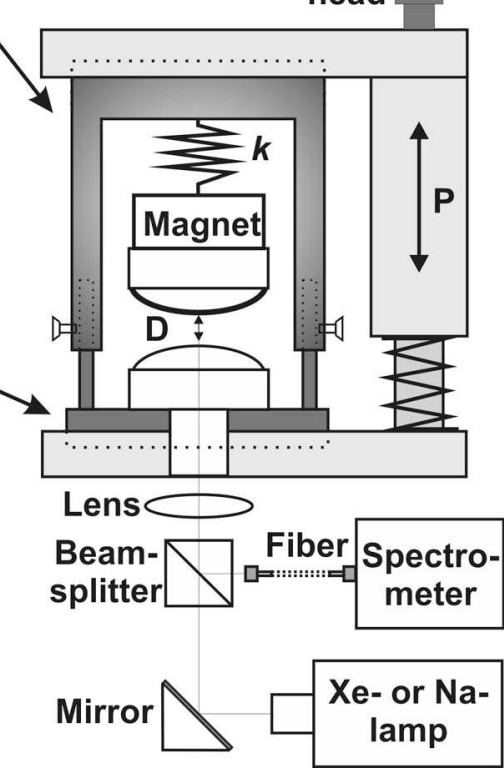

FIG. 1. eSFA and pre-adjustment. (a) The miniature eSFA consists two parts that can be positioned and fixed relative to each other. The upper surface and the permanent magnet are connected to the upper part via a helical spring. The other surface is rigidly mounted to the bottom part. The back-silvered mica surfaces form a Fabry-Pérot interferometer. The manual pre-adjusting aid is shown in (b). Via a micrometer head the surface separation is manually adjusted to a few micrometers while the interference pattern is analyzed with a spectrograph. (c) The point of closest approach (PCA) can readily be identified using Newton rings.

\section{A. The miniature eSFA}

We designed a miniature stainless steel eSFA to embrace the two cylindrically shaped glass substrates (BK7, Crylight Photonics, China) which act as holders for the mica surfaces in the experiment. The upper glass substrate is rigidly connected to a steel tube that embodies a permanent magnet (NdFeB, Webcraft GmbH, Switzerland). A helical spring (Durovis, Switzerland) with spring constant $\mathrm{k}=1750 \pm 70 \mathrm{~N} / \mathrm{m}$ connects the upper surface including the magnets to the upper eSFA part. It is laser soldered to both the magnet tube and to the upper part of the miniature eSFA. The lower part consists of an optically blackened steel socket with three pressfitted dowel pins; it centers the second glass substrate. At a nominal surface separation of $\mathrm{D} \approx 3-5 \mu \mathrm{m}$ six headless $\mathrm{M} 2$ screws can be tightened to lock the two eSFA parts in place. The hence pre-adjusted eSFA is then gently transferred into the autoclave. The pre-adjusting and the autoclave transfer are completed inside a laminar air flow cabinet to prevent particle or dust contamination. To assure optimal stability of surface separation during pressure variations the eSFA rests in the autoclave with a single point contact, which is at the optical window.

\section{B. Two stage surface separation control}

To control surface separation we utilize two stages. The coarse stage is designed to set an initial surface separation of a few micrometers. The finer stage then uses a magnetic drive.

The coarse approach stage is based on an external auxiliary pre-adjustment tool. This auxiliary device uses a micrometer head (Series 153-101, Mitutoyo, Switzerland) for manual displacement of the two parts relative to each other. To keep the exact same optical light path, the auxiliary device temporarily occupies the place of the autoclave. This practice allows one to use the same optical distance measurement during pre-adjustment as during experiments. The surface separation is measured and displayed on a computer screen with refresh rates of a few $\mathrm{Hz}$. With this distance monitoring it is straightforward to position the two eSFA parts at a selected surface separation to within a few $100 \mathrm{~nm}$ before tightening the locking screws.

As described above a permanent magnet is attached to the upper surface. A low-resistivity copper coil, which is wound around the autoclave, delivers a current-induced magnetic field gradient into the autoclave. A computer controlled (UTA 12, EA Elektro-Automatik, Germany) and stabilized current source (PS 3016-20B, EA Elektro-Automatik, Germany) is used to drive that coil. The dimensions of the coil as well as the power supply are chosen such that a minimal distance resolution of $0.1 \mathrm{~nm} / \mathrm{bit}$ and a maximal power dissipation of $0.7 \mathrm{~W}$ at full deflection are guaranteed. The magnet drive has a bipolar operating range of $14 \mu \mathrm{m}$. We developed a LABVIEW subroutine that continuously runs in the background to control all basic functions (positioning, speed, limits, etc.). Additionally, we found it useful to have a secondary coil integrated into the optical table to bias the magnetic drive during initial pre-adjustment and autoclave transfer. 


\section{The optics setup}

To perform thin film interferometry in reflection we built a three-level optical stand that provides a recess on the top (Fig. 1(b)) either for the pre-adjustment device with eSFA or, alternatively, for the autoclave with eSFA. The top level of the stand also holds a three-axis adjustable bi-convex focusing lens (Edmund optics, Germany). The mid-plane houses a non-polarizing cube beam splitter (Edmund optics, Germany) as well as the miniature spectrograph (USB2000+, Ocean optics, USA). A monochrome imaging camera (DVC 4000 , DVC company, USA) is optionally used to capture the contact area. Aluminum coated $45^{\circ}$ tilt mirror (Edmund optics, Germany) is located on the lower level (Fig. 1(b)), where also the light sources are coupled in. All structural parts near the path of light are blackened to reduce unwanted reflections.

In the experiment light is analyzed from the point of closest approach (PCA). To facilitate initial centering of the optics to this PCA we use illumination by a low pressure sodiumvapour lamp (Elevite, Switzerland). The sodium lamp emits two dominant spectral lines (589.0 and $598.6 \mathrm{~nm}$ ) and exhibits a large coherence length of roughly $15 \mathrm{~mm}$, which is particularly useful to visualize so called Newton rings at surface separations $>200 \mu \mathrm{m}$. Newton rings are captured with the imaging camera (Fig. 1(c)). Alignment of the optics at the PCA and setting an initial surface separation of $10 \mu \mathrm{m}$ is straightforward in this configuration. We then switch to a white light source (xenon arc lamp, Müller, Germany) for illumination of the spectrograph, which will detect the interference fringes in Fourier space for the high precision measurement. The cubic beam splitter can therefore be rotated by $90^{\circ}$ in reproducible fashion using a rotation stage (RSP-2T, Newport, Germany). Furthermore, an XY translation stage (MT1-Z8, Thorlabs, USA) is used to center the end of a $200 \mu \mathrm{m}$ diameter optical fiber (Ocean optics, USA) onto the virtual image of the PCA (Fig. 1(b)). The eSFA is equipped for the fast spectral correlation (FSC) method that allows real time spectral analysis and monitoring of surface separation for further fine adjustments as well as the final experiment.

\section{The autoclave}

Illustrated in Fig. 2, the autoclave is a titanium container that tightly fits around the eSFA for minimal pressurized fluid volume. The autoclave has a cylindrical base shape with a flange at one end (bottom) and an electrical pressure sensor (Series $8201 \mathrm{H}$, MTS, Switzerland) at the other end (top). An optical window (BK7, Crylight Photonics, China) is fitted into the flange. The sealing is established using viton O-Ring gaskets at the side faces of both flange and window. A recess in the flange prevents out-sliding of the window under pressure. During operation, and also during pre-positioning, white-light is directed through the same optical window to assure invariant optical path.

The lower glass substrate of the miniature eSFA is slightly protruding $(0.1 \mathrm{~mm})$ and forms a single rigid contact with the also slightly protruding optical window of the

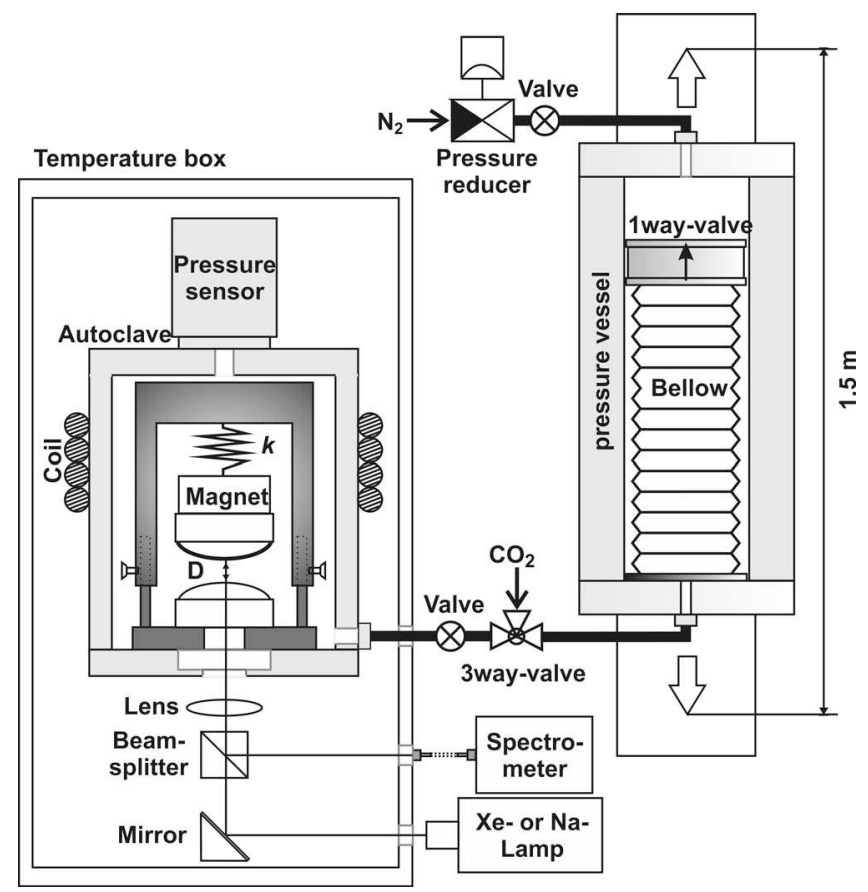

FIG. 2. The measurement setup. The eSFA is placed inside an autoclave (left). A copper coil is wound around the autoclave. A current can be used to deflect the upper surface. Steel tubing connects the autoclave with a pressure vessel (right). It contains a metal bellow for $\mathrm{N}_{2}$ pressurization. A one-way-valve limits the bellows pressure. An electrical pressure reducer controls the pressure in the $\mathrm{N}_{2}$ circuit. For fine adjustments ( \pm 0.1 bar), the pressure vessel can be vertically displaced by means of a motorized translation stage.

autoclave flange. The eSFA is gently held against this rigid contact via a small spring load from the other side of the autoclave (not shown).

The autoclave is fitted with an inlet and an outlet to connect the feed and the exhaust of the pressurized fluids.

\section{E. The pressure control}

To control the pressure of the fluid a separate pressure control system is used. The here used medium $\mathrm{CO}_{2}$ is special inasmuch as the pressure inside a carbon dioxide bottle is independent of filling and is 58-62 bars at ambient because there is coexistence of liquid and gas phases inside the bottle. Performing measurements at elevated pressures, i.e., up to 170 bars, calls for a contamination-free $\mathrm{CO}_{2}$ compressor, which is non-standard. Therefore, we welded a metal bellow (Series 245-15, Huntington, USA) with a nominal volume of $0.26 \mathrm{dm}^{3}$ into a flange that fits onto a cylindrical pressure vessel. At the free end, which will be inside the pressurized vessel, we laser-soldered a one-way exhaust valve (RK 86a, Ramseyer, Switzerland) to the bellow that can open at a pre-determined overpressure of 0.1 bar. This bellowflange is finally slid into the cylindrical pressure vessel. The top flange of the pressure vessel is connected via a $1 / 8^{\prime \prime}$ stainless steel tube (Swagelok, USA) to a 200 bars nitrogen bottle equipped with an automated electro-pneumatic reducing device (ER 3000, Tescom, Germany). A three-way valve 
connects the bottom flange of the pressure vessel with the carbon dioxide circuit including $\mathrm{CO}_{2}$ bottle and the eSFA autoclave. Prior to filling, the whole system can be evacuated by a membrane pump (MVP 015-4, Pfeiffer Vacuum, Germany) to a nominal pressure of a few millibar. With the eSFA autoclave venting faintly open one can thoroughly purge the system with gaseous carbon dioxide $(99.99 \%$, Carbagas, Switzerland). After purging, the vent can be closed and liquid carbon dioxide is slowly transferred from the $\mathrm{CO}_{2}$ bottle into the pressure vessel under gas-liquid coexistence conditions. To facilitate condensation in the $\mathrm{CO}_{2}$ circuit, we have placed the $\mathrm{CO}_{2}$ bottle with a dive tube one floor (i.e., $\approx 3.5 \mathrm{~m}$ ) above the eSFA laboratory. To determine the level of filling, the vessel is freely suspended on a strain gage (XMB, MTS, Switzerland) balance. The half-bridge gage is read-out via a homebuilt amplifier. The mass of the liquid carbon dioxide inside the vessel is determined to a precision of $5 \mathrm{~g}$. The maximum filling is about $200 \mathrm{~g}$. During filling, some $\mathrm{CO}_{2}$ inevitably exits through the one-way valve on top of the bellow for pressure equilibration. The pressure in the $\mathrm{N}_{2}$ circuit (i.e., outside the $\mathrm{CO}_{2}$ bellow) can be monitored with a mechanical manometer. Simultaneously the electrical pressure sensor on top of the eSFA autoclave is indicating the pressure in the $\mathrm{CO}_{2}$ circuit. This electrical pressure sensor signal can also be used as feedback to the electro-pneumatic reducing device controlling the pressure in the $\mathrm{N}_{2}$ circuit. After closing the $\mathrm{CO}_{2}$ feed from the bottle, the nitrogen is used to further compress the carbon dioxide inside the bellow. We developed a LABVIEW software to control the automated reducing device in a fine pulse mode, which limits the pressure rates in the system to about $1 \mathrm{bar} / \mathrm{min}$. If the feedback loop was set to PID controlled mode the pressure rates can reach $120 \mathrm{bar} / \mathrm{min}$, which would be detrimental for our gaskets. Namely, at pressure drop rates of more than some $10 \mathrm{bar} / \mathrm{min}$ the viton gaskets are irreversibly damaged by dissolved gas expansion.

A fine-control of pressure is realized using gravity differences. Therefore, the pressure vessel is suspended on a vertical translation stage (EP 1820, Phoenix mecano, Germany). The driving spindle is coupled to a DC-minimotor (Series 2342, Faulhaber, Switzerland) with a planetary gear (Series 23/1, 0.7 Nm torque) and a magnetic impulse generator (512 impulses per revolution) for position control. The minimotor is controlled via a computer card (DCX-PCI 100, PMC corp., USA). The total vertical range of displacement is $1.5 \mathrm{~m}$. This height difference results in a pressure change of 0.1 bar, in liquid $\mathrm{CO}_{2}$, as measured in the stationary eSFA autoclave while connected to the system. The pressure vessel and the vertical translation stage are individually enclosed in a polystyrene thermal insulation box, which is equipped with a 320 Watt Peltier thermal heat exchanger.

A special solution had to be found for the tubing, which connects the eSFA autoclave with the pressure vessel. The connectors at the autoclave are reusable Swagelok VCR fittings, whereas the pressure vessel is equipped with fixed Swagelok ferrule fittings. At two locations we used Loctite 577-a dimetacrylate ester as a sealant; namely for the VCR fittings on the autoclave and to seal the manometer on the pressure vessel.

\section{F. The temperature control}

The thermal control of both eSFA autoclave and pressure vessel are realized using individual Peltier heat exchangers as described previously. ${ }^{11}$ The temperature range of the eSFA autoclave is -20 to $60{ }^{\circ} \mathrm{C}$ with a stability of $0.002{ }^{\circ} \mathrm{C} / \mathrm{h}$. The thermal insulation box surrounding the eSFA autoclave and the optical stand is posed on a vibration damped optical table (Integrated Dynamics Engineering, Germany).

\section{RESULTS}

\section{A. Equilibration and stability}

Operating the pressurization is a semi-automated process. After evacuating the autoclave we reconnect it to the pressure vessel (i.e., $\mathrm{CO}_{2}$ circuit) by gently opening a needle valve ensuring pressure rates of less than $1 \mathrm{bar} / \mathrm{min}$. In Fig. 3 we show the pressure and the surface separation as functions of time during such a filling process of the autoclave. The temperature control is OFF at this time, but the temperature inside the laboratory in the basement is rather stable at $21 \pm 1{ }^{\circ} \mathrm{C}$. We note that a stepwise pressure increase from 3 to 58 bars entails initial drifts of surface separation at rates of $50 \pm 10 \mathrm{~nm} / \mathrm{bar}$. At 58 bars the pressure in the autoclave is again in equilibrium with the pressure vessel. To further increase the pressure we use the electro-pneumatic reducing device on the nitrogen side. It is interesting to note that at 63.3 bars the surface separation increases abruptly by $9 \pm 1 \mu \mathrm{m}$. This can be used as a clear indicator for liquid condensation around the eSFA. The upper spring suspended surface is now entirely immersed in the liquid phase. As expected, the refractive index measured interferometrically between the surfaces changes abruptly from 1.05 to 1.18 . We note that the observed spring deflection of $9 \pm 1 \mu \mathrm{m}$ corresponds to a force of $16 \pm 2 \mathrm{mN}$, which is in the order of

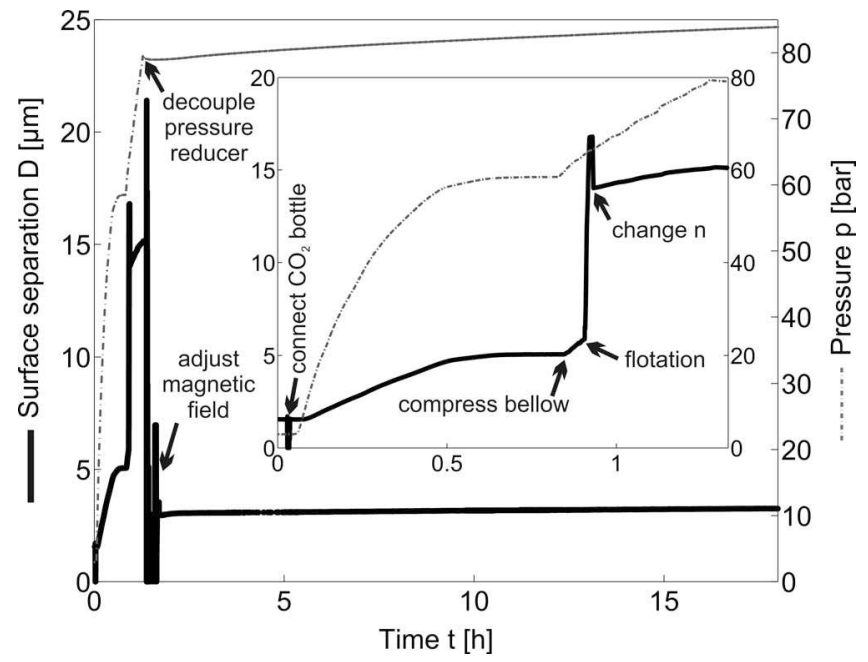

FIG. 3. Effect on surface separation. The surface separation, D, (thick black curve) and the pressure, p, (thin dotted curve) are plotted as a function of time during a filling process. Notable changes in experimental conditions are highlighted by arrows. The inset is a zoom-in of the first hour. It illustrates the flotation of the upper surface upon gas-liquid transition. After the flotation we have changed the refractive index, n, manually, because at that time the refractive index library was not yet included in the evaluation software. 


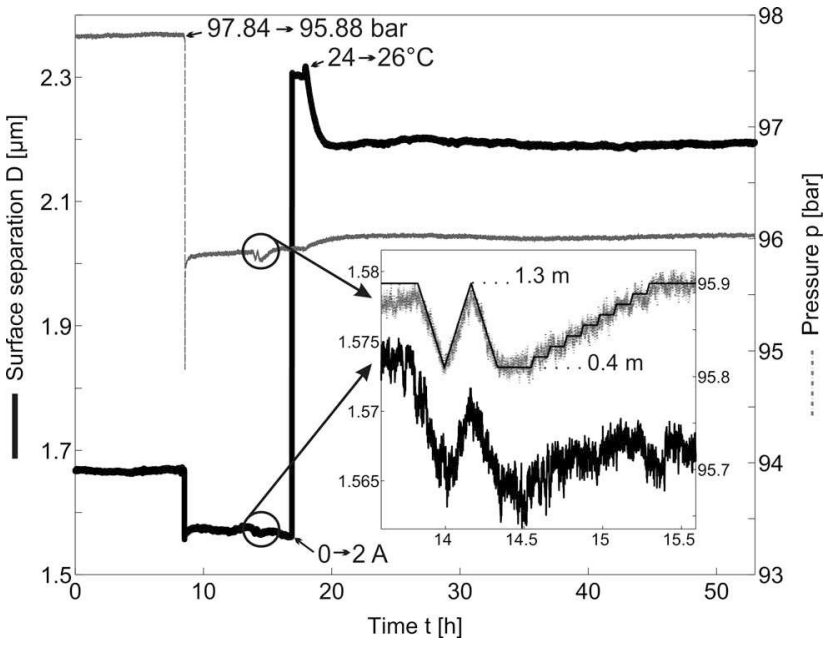

FIG. 4. Instrumental drift. The surface separation, D, (black curve) and the pressure, p, (dotted gray curve) are plotted against time while changing different control parameters. The starting times are highlighted with arrows. The inset shows a magnification of the region of interest where the vertical fine pressure control was tested. The vessel was vertically displaced by $0.9 \mathrm{~m}$. For illustration of this fine control, the noise-free black curve superimposed to the measured pressure (gray) in the inset represents the motor position (scale $0.4 \mathrm{~m}$ to $1.3 \mathrm{~m})$.

the expected Archimedes floatation force of the upper surface (the density of liquid carbon dioxide at $22.8^{\circ} \mathrm{C}$ and 64.0 bars is $0.73 \mathrm{~kg} / \mathrm{dm}^{3}$ (Ref. 12) and the volume of the immersed body measures $3.2 \pm 0.3 \mathrm{~cm}^{3}$ ).

After further compressing the carbon dioxide with nitrogen to 78 bars, we close a valve to isolate the $\mathrm{N}_{2}$ circuit from active pressure regulation. During a first $10 \mathrm{~h}$ after this separation of the system the pressure drift is up to $280 \pm 50 \mathrm{mbar} / \mathrm{h}$. After 1 week of equilibration this value decreases to typically $50 \mathrm{mbar} / \mathrm{h}$. And after 2 weeks we have reached a steady-state around 100 bars with drift rates of less than $2 \mathrm{mbar} / \mathrm{h}$. Due to the expansion and compression of the different media in the system upon initial filling and compression, and, due to the relatively large thermal mass of the pressure vessel, we create temperature gradients of a few $\mathrm{K}$, which cause the mentioned pressure drifts.

In Fig. 4 we illustrate the equilibrated response of the system to a change of selected parameters (pressure, temperature, magnetic coil current). The surfaces are initially at an equilibrium separation, $\mathrm{D} \approx 1.6 \mu \mathrm{m}$. The temperature stability inside the polystyrene box is better than $2 \mathrm{mK} / \mathrm{h}$. The pressure stability is better than $2 \mathrm{mbar} / \mathrm{h}$ (with a $7 \mathrm{mbar}$ measurement noise). A stability of the surface separation on the order of $\mathrm{dD} / \mathrm{dt} \approx 10 \mathrm{pm} / \mathrm{min}$ can be achieved, which is comparable to the stability of the conventional eSFA at ambient.

If we decrease the pressure by 2 bars at $\mathrm{t}=8.5 \mathrm{~h}$ the surface separation decreases by $0.09 \mu \mathrm{m}$ in response (45 nm/bar). This pressure reduction step entails again some temperature gradients in the system and the pressure drift rate momentarily increases to $8 \mathrm{mbar} / \mathrm{h}$. After some equilibration, we induce a fine pressure change at $\mathrm{t}=14 \mathrm{~h}$ by moving the pressure vessel vertically over a total height of $\Delta h=0.9 \mathrm{~m}$ (Fig. 4 and inset). Each vertical displacement of $0.1 \mathrm{~m}$ results in a pressure increase of 10 mbar. The change in pres- sure again has an influence on the surface separation due to flotation forces.

At time $\mathrm{t}=17 \mathrm{~h}$ we actuate the upper surface by changing the coil current from 0 to $2 \mathrm{~A}$. This immediately changes the surface separation from 1.56 to $2.32 \mu \mathrm{m}$. At a current of $2 \mathrm{~A}$ the power dissipation of the magnetic coil is $7 \mathrm{~mW}$. The system proceeds to the new steady state; temporarily exhibiting a small increase of pressure drift rate, which remains, however, below $0.7 \mathrm{mbar} / \mathrm{min}$.

In another isochoric step at $\mathrm{t}=18 \mathrm{~h}$ we change the temperature set point in the thermal insulation box from $24^{\circ} \mathrm{C}$ to $26{ }^{\circ} \mathrm{C}$. The issued increase of $2{ }^{\circ} \mathrm{C}$, again, temporarily causes a moderate pressure drift rate increase of $1.8 \mathrm{mbar} / \mathrm{min}$. After $150 \mathrm{~min}$ the system reaches a new steady state with pressure drift rates as low as $0.03 \mathrm{mbar} / \mathrm{min}$. The surface separation responds at a ratio of $60 \mathrm{~nm} /{ }^{\circ} \mathrm{C}$ and the autoclave pressure at $70 \mathrm{mbar} /{ }^{\circ} \mathrm{C}$ to controlled temperature changes. It is important to notice that this prominent relationship between temperature and autoclave pressure means that any thermal drift always implies a pressure drift, which itself has an influence on the surface separation (i.e., flotation). Temperature changes are thus the prime source of drifts in the sealed system and therefore determine the practical equilibration times.

As another potential source of drift, we have also checked the stability of our current source (i.e., coil current) to be less than $12 \mu \mathrm{A} / \mathrm{min}$ which is equal to a change in distance of $5 \mathrm{pm} / \mathrm{min}$ and thus negligible in all previous considerations.

\section{B. Measuring surface forces in $\mathrm{CO}_{2}$}

The interferometric measurement of most SFAs is configured in transmission. ${ }^{1,13-16}$ The here presented instrument uses reflection to reduce the number of sealed windows in the autoclave. The two equally thick muscovite mica sheets (each $2-5 \mu \mathrm{m}$ ) are now prepared with different silver mirrors, namely $25 \mathrm{~nm}$ and $160 \mathrm{~nm}$. The mica sheet with $160 \mathrm{~nm}$ silver is more reflective and is thus placed at the upper sample holder in the eSFA. Together, the two surfaces form a Fabry-Pérot interferometer in analogy to the transmission configuration. The resulting interference pattern is, however, destructive and exhibits extinction fringes instead of interference peaks. This has some important practical consequences as we shall see. In Fig. 5 a sample white-light corrected spectrum is plotted together with the corresponding theoretically predicted one. It is a situation where the two surfaces are in contact. The inset shows two selected extinction fringes of odd and even order. We note already how asymmetric they are and that there is only a $\approx 30 \%$ intensity modulation in the spectrum. The multi-layer matrix method can also be used to calculate the theoretical reflectivity coefficient of such thin film interferometer. Therefore, it was appropriate to adopt the existing FSC evaluation ${ }^{17}$ with only a few minor software changes. The precision (i.e., standard deviation) of surface separation measurement in reflection is $\pm 30 \mathrm{pm}$ and therefore comparable to the established transmission setup. The acquisition rates achieved at this resolution is $\approx 2 \mathrm{~Hz}$.

To demonstrate the full function of the pressurized eSFA we performed surface force measurements at a temperature 


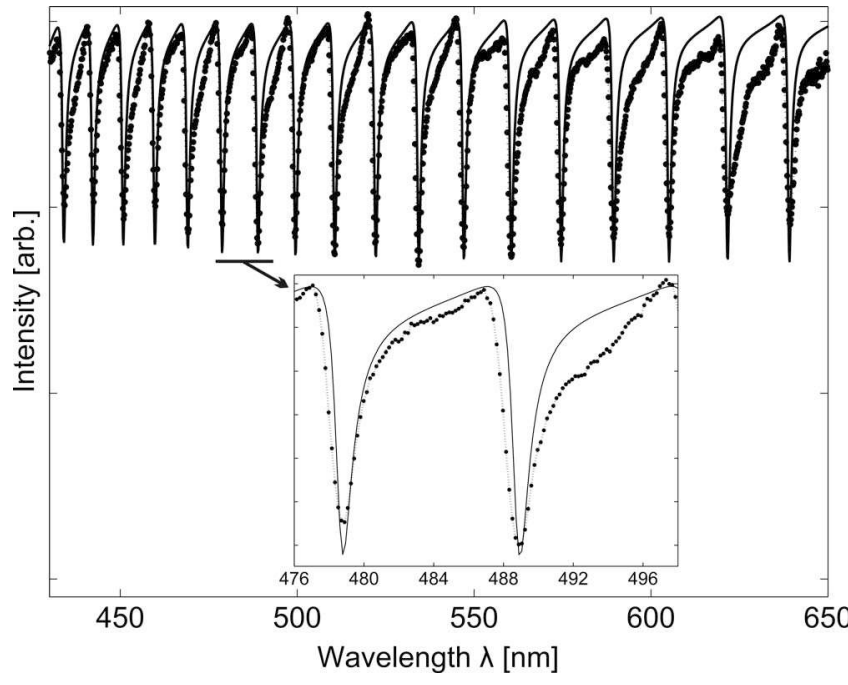

FIG. 5. Interference spectrum in reflection. The solid black line shows the theoretical spectrum of a 3-layer etalon $(160 \mathrm{~nm}$ silver, $10.4449 \mu \mathrm{m}$ muscovite mica, $25 \mathrm{~nm}$ silver). The points correspond to the measured spectrum. The inset shows a detailed view of the shape of odd and even chromatic order extinctions.

of $27 \pm 0.5{ }^{\circ} \mathrm{C}$. The resulting force-distance curves are displayed in Fig. 6. The standard procedure is to continuously reduce surface separation starting at $1.0 \mu \mathrm{m}$ using the magnet drive at a speed of $1 \mathrm{~nm} / \mathrm{s}$. After surface contact, we continue increasing the magnetic loading force to $5 \mathrm{mN}$, which would correspond to a nominal free motion of additional $-3.0 \mu \mathrm{m}$. The same procedure is repeated at different static pressures, namely $\mathrm{p} 1=0.3, \mathrm{p} 2=1.0, \mathrm{p} 3$ $=10.5$, and $\mathrm{p} 4=73.6$ bars, respectively, as shown

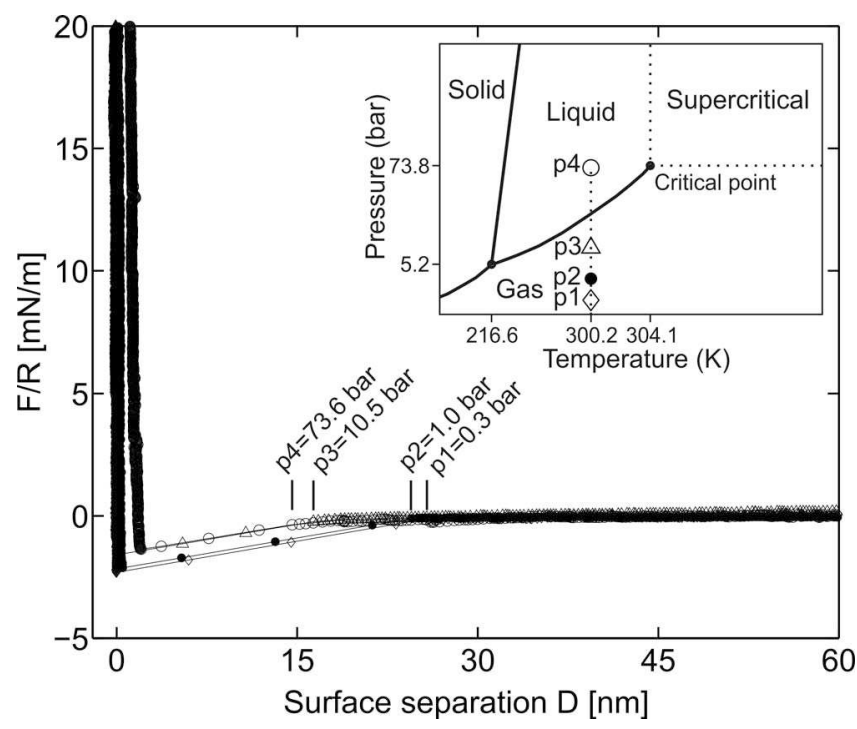

FIG. 6. Force-distance measurements in carbon dioxide. We measure the surface force (normalized by cylinder radius, $\mathrm{R}$ ) while steadily decreasing the surface separation. We repeated this procedure at four different pressures at $300.2 \mathrm{~K}$. The spring instability occurs at different surface separations due to variable viscous vibration damping in the fluid (i.e., $25 \mathrm{~nm} @ 0.3 \mathrm{bar}$ and $14 \mathrm{~nm} @ 73.6$ bars). The 73.6 bars curve shows that the surfaces are separated by molecularly layered $\mathrm{CO}_{2}$ after the jump. Similar layering is known to occur in other simple liquids. ${ }^{22-24}$ A detailed discussion is, however, outside the scope of this paper. in Fig. 6 inset. The pressure drift rates throughout these four measurements are smaller than $3 \mathrm{mbar} / \mathrm{min}$. We observe surface forces of Van der Waals type with a jump into contact. At 1 bar we measure a jump-in-distance of 23 $\pm 2 \mathrm{~nm}$, which is comparable to other SFAs operated in a nitrogen environment at ambient conditions. As soon as we pressurize the fluid becomes more viscose and vibrationinduced surface oscillations are damped. In addition the permittivity of the medium is higher, which decreases the Van der Waals interactions accordingly - altogether leading to smaller jump distances (e.g., $13 \pm 2 \mathrm{~nm} @ 73.6$ bars) for the spring instability. ${ }^{18}$

The spring instability condition, $\mathrm{dW} / \mathrm{dD} \geq \mathrm{k}$, determines the surface distance at which the jump occurs. In the case of 73.6 bars-in liquid $\mathrm{CO}_{2}$ - the calculated jump-in-distance, in the absence of vibrations, would be $4.2 \mathrm{~nm}$. The here measured jump-in-distances are $9 \pm 2 \mathrm{~nm}$ larger. We interpret this difference as the maximum amplitude of the vibrationinduced surface motion. Our main sources for mechanical vibrations are the ventilator of the temperature box, the electrical and optical cables connections between the anti-vibration table and the infrastructure, namely the rigid steel tube connecting the eSFA with the pressure vessel. To effectively suppress vibration coupling we damped all the power and measuring lines as well as the steel tube using pairs of heavy brackets with soft foam linings-one bracket on the antivibration table with its counterpart on a normal table.

\section{DISCUSSION}

In contrast to an interferometric measurement in transmission where equal semi-transparent mirrors are used, the mirror properties must be different for reflection optics. Ideally, one surface is totally reflecting and the other is semitransparent. To find the optimal silver thickness, we calculated spectral reflectivities for the thin film interferometer using the matrix multilayer method. Figure 7 inset (a) illustrates how a particular extinction fringe theoretically changes as the semitransparent mirror thickness is varied. If the reflective surface is covered with a silver mirror thicker than $100 \mathrm{~nm}$, the shape of the extinctions remains constant. We adopted the practice of using $160 \mathrm{~nm}$ thickness for the reflective mirror. For easy machine detection of fringe position, we optimize for spectral contrast. Figure 7(a) shows how even small changes in the silver thickness of the transmitting mirror can have a significant influence on spectral contrast. An optimum is obtained around $25 \mathrm{~nm}$ silver thickness. We have experimentally verified and fine-tuned this theoretical prediction by using mirror thicknesses of 100, 130, and $160 \mathrm{~nm}$ for the reflective mirror and 20, 25, and $30 \mathrm{~nm}$ for the semi-transparent mirror.

It is important to note that the optical properties of a $25 \mathrm{~nm}$ silver mirror are particularly sensitive to oxidation or contamination. Figure 7(b) compares a simulated and a measured spectrum with slight oxidation. The shape of the measured spectrum is immediately broadened due to minimal oxidation (not visible to the eye). An XPS analysis of the surface revealed contaminations of oxygen, carbon, sulfur, and chloride in a range of only a few atomic percent. Special care must 


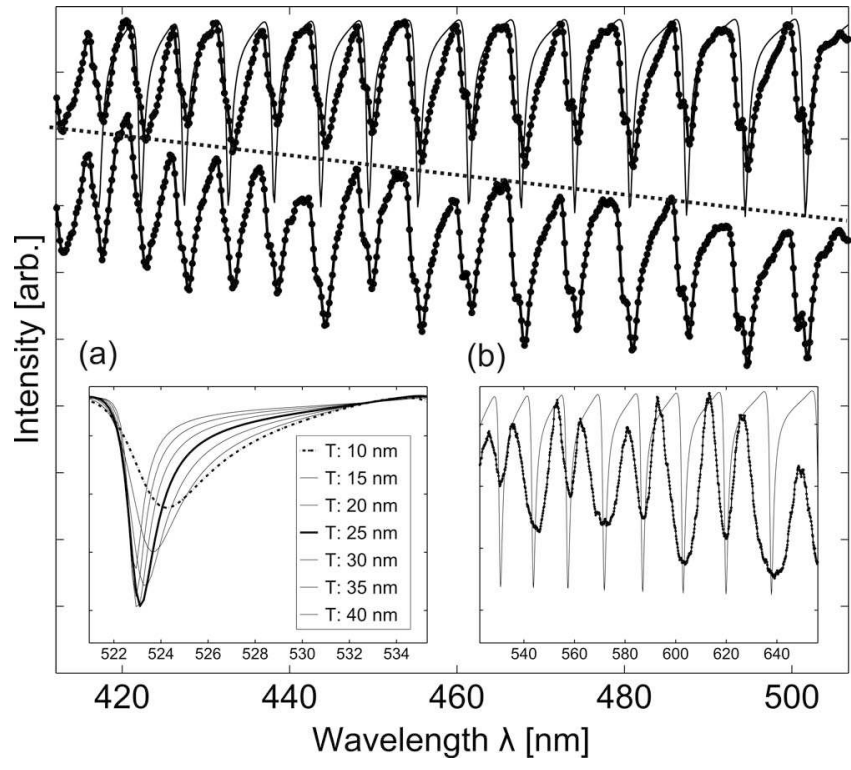

FIG. 7. Optimization of reflection spectrum. After a white-light correction the measured spectrum has a flat baseline (top black points) and fits well the simulated spectrum (top black curve). If one of the optical elements in the light path changes slightly its position, the baseline of the spectrum is different (dotted slope). Inset (a) illustrates theoretical spectra for an etalon with variable transmission mirror thickness at fixed reflection mirror thickness (160 nm silver). Optimal spectral contrast is obtained around $25 \mathrm{~nm}$ (black curve). Inset (b) illustrates the measured reflection spectrum (black points) after a minor oxidation of the transmission mirror as compared to the theoretical spectrum (black curve)

be taken during storage of these thin mirrors prior to use in the eSFA.

As one might expect, measuring extinctions in a reflected interference spectrum is technically more challenging than measuring the peaks of a transmission spectrum. This is due to the fact that exact fringe positions now have to be determined at the weakest intensities in the spectrum. Low intensities are more affected by shot noise, backscattering of light, and unwanted illumination from diffuse stray light. Parasitic light can originate from any light scattering objects near the path of light. We therefore had to carefully blacken all metal parts near the optical path. Introducing several aperture stops also proved to be helpful. We note that scattered light can have another spectral density (i.e., color) than the incident light. In practice, this even applies to technically black metal surfaces. Since the optical path is slightly altered during all optical alignment it is valuable to determine the optical transmission function (i.e., white light correction) after alignment. This can significantly improve the correct detection of spectral extinctions. Therefore, we record and average the interference spectrum (incl. interference extinctions) several hundred times while the surface separation is continuously changed with the magnetic drive over a distance of at least $1 / 4$ wavelengths. The extinction fringes are thus shifted at least by $2 \pi$ in the phase space and consequently averaged out. Division of all subsequently measured spectra by this average transmission function finally mimics ideal white light illumination as required for high precision FSC evaluation.

Although the FSC evaluation algorithm allows independent determination of refractive index and surface separation, it is more robust against detection noise if a good starting point for the refractive index is provided. For this purpose, we have included the dispersive refractive index data from Ref. 19 into the software refractive index library. Therein, Michels et al. measured the change in refractive index, n, of $\mathrm{CO}_{2}$ under different pressures, $\mathrm{p}$, at six different wavelengths, $\lambda$, and four different temperatures, $\mathrm{T}$. We had to extrapolate each $\mathrm{n}(\mathrm{p}, \lambda)$-surface of this data to our measurement window. Using Cauchy's formula $\mathrm{n}(\lambda) \approx \mathrm{A}+\mathrm{B} / \lambda^{2},{ }^{20}$ we can extract two coefficient-matrices $\mathrm{A}(\mathrm{p}, \mathrm{T})$ and $\mathrm{B}(\mathrm{p}, \mathrm{T})$ for any given pressure and temperature.

Besides magnetic actuation, the surface separation, measured by interferometry, is also affected by temperature and pressure (see Sec. III). When the surfaces are not in contact, the recorded surface separation is a measure for the drift stability of the instrument's mechanical loop. To this end we quantify and compare the most important effects on surface separation:

(i) The silvered mica surfaces are glued with a layer of EPON 1004 thermoset resin (Young's modulus: 2-2.2 GPa) to the cylindrical glass substrates. The thickness of the glue is in the range of $20-30 \mu \mathrm{m}$. We quantify a compression of the glue of $<0.3 \mathrm{~nm} / \mathrm{bar}$.

(ii) The other elements in the mechanical loop are of BK7glass and metal with Young's modulus contrast of 81 $\mathrm{GPa}$ and $200 \mathrm{GPa}$, respectively. The dilation of the mechanical loop due to maximal design pressures is thus expected $<10 \mathrm{~nm} / \mathrm{bar}$.

(iii) In addition to the compressibility we also have thermal expansion of each part in the mechanical loop. We have measured that the equilibrium thermal effect is in the order of $60 \mathrm{~nm} /{ }^{\circ} \mathrm{C}$.

(iv) Another effect is related to the change of flotation due to variations of density in the fluid. The upper surface that is suspended on the $\mathrm{k}=1.7 \mathrm{kN} / \mathrm{m}$ spring displaces a design volume of $3.2 \pm 0.2 \mathrm{~cm}^{3}$; i.e., including permanent magnet, the BK7 glass substrate and the metal holders. An exemplary pressure change from 65 to 85 bars at $25^{\circ} \mathrm{C}$ entails a liquid $\mathrm{CO}_{2}$ density change increase from 0.71 to $0.79 \mathrm{~kg} / \mathrm{dm}^{3}$. In need of extrapolation from existing data, these densities are calculated with the Lorentz-Lorenz formula using the data of refractive indices from Ref. 19 The flotation induced dilation of the mechanical loop is in the order of $50 \pm 10 \mathrm{~nm} / \mathrm{bar}$. We recall that this floatation effect is of practical use, since it allows easy detection of the gas-liquid transition in the eSFA.

(v) Obviously, the surface separation is also affected by parasitic magnetic fields. Such fields can be generated by possibly magnetized parts (autoclave, tools, etc.) in the vicinity of the eSFA. We have come to use ceramic tools and a titanium autoclave to minimize the effect. (We learned the lesson that even nonmagnetic steel can magnetize when machined upon) There are some inevitable small magnetic fields from steel screws, optical holders, and the pressure sensor. Quantifying these static offsets is, to some extent, practically useful; for example, if the surface 
separation is known to decrease by $7 \mu \mathrm{m}$ when sliding the eSFA into the autoclave, the pre-adjustment must be set to about $10 \mu \mathrm{m}$ to finally reach the desired $3 \mu \mathrm{m}$.

Medium leakage is one of the prime causes of pressure drifts. The test is yet simple. If we disconnect the autoclave from the pressure vessel we measure leak rates of $30-150 \mathrm{mbar} / \mathrm{h}$. Leaking rates below this range are difficult to achieve with a reconnectable autoclave. We can, however, significantly improve the situation if we keep the whole system connected. Due to the much larger volume the pressure drift reduces to $<2 \mathrm{mbar} / \mathrm{h}$.

Measuring structural forces in a fluid calls for a fluid purity. We cleaned each individual part several times in an ultrasonic ethanol path, rinsed it with "milli-q" water and dried it with pure nitrogen gas. To connect the tubing we use high-purity, high-pressure VCR diaphragm valves, and nonlubricated Swagelok fittings with a two-ferrule mechanism. We use Loctite to seal the two threads at the autoclave and the pressure vessel. In the first tens of filling cycles these residues were dissolved and rinsed out by the liquid carbon dioxide. We have finally used more than 50 rinsing cycles to assure clean $\mathrm{CO}_{2}$ in the system.

The carbon dioxide may also be contaminated by $\mathrm{N}_{2}$. The carbon dioxide and $\mathrm{N}_{2}$ circuits are separated by the metal bellow and the integrated one-way valve. We verified this possible source of $\mathrm{N}_{2}$ contamination using gas chromatography. The amount of nitrogen found in carbon dioxide pressurized at 95 bars for weeks was only 1.6-5.1 times higher than the amount found in the reference gas taken directly from the $\mathrm{CO}_{2}$ bottle $(99.99 \%)$. We conclude that the amount of nitrogen dissolved in carbon dioxide in our pressurized system is $<0.1 \%$, which we consider acceptable.

The here described eSFA with pressure control can be used in a wide range of novel experimental work in the future. Notably, this includes the study of different important fluids with convenient phase diagrams like $\mathrm{CO}_{2}$, ethane, propane, ethylene, propylene, degassed water, methane, or neat oils.

In the case of $\mathrm{CO}_{2}$, a forthcoming publication will study the molecular structure in nanometer confined liquid $\mathrm{CO}_{2}$ films. In supercritical $\mathrm{CO}_{2}$ it is of fundamental interest to test the theory of dispersive surface forces by gradually changing medium density and permittivity. Near the critical point of $\mathrm{CO}_{2}$ we finally hope to detect the thermodynamic equivalent of the Casimir force ${ }^{21}$ as well as optically detect near-critical opalescence in the interference spectrum due to extended density fluctuations.

Determining interfacial viscosities by squeeze experiments under hydrostatic pressures is expected to yield a wealth of information that is useful in the scientific fields of tribology or geology. The fact that the autoclave is well sealed also provides the opportunity to study neat degassed fluids at any locus of their phase diagram. For example, we can now study the role of dissolved gasses on the hydrophobic interaction in great detail across aqueous solution, which is still a controversial topic.

\section{ACKNOWLEDGMENTS}

We thank Daniel Rechenmacher (Empa) and Joerg Gschwend (Empa) for their support and expertise in designing, engineering and the production of the instrument parts. Janine Sueess (Empa) and Jaroslav Vanicek (ETH Zürich) have helped us to set up thermal insulations and the temperature control. We are also indebted to Cornelia Seiler (Empa) for gas impurity analysis using gas chromatography. Furthermore, we are grateful to Nicholas Spencer (ETH Zürich) for fruitful scientific discussions. This work was supported by the Swiss National Science Foundation.

${ }^{1}$ J. N. Israelachvili and G. E. Adams, J. Chem. Soc., Faraday Trans. 74, 975 (1978).

${ }^{2}$ A. Scheludko, Kolloid-Z. 155(1), 39 (1957).

${ }^{3}$ B. L. Pruitt and T. W. Kenny, Sens. Actuators A 104(1), 68 (2003).

${ }^{4}$ S. C. Clark, J. Y. Walz, and W. A. Ducker, Langmuir 20(18), 7616 (2004).

${ }^{5}$ R. Tykhoniuk, J. Tomas, S. Luding, M. Kappl, L. Heim, and H. J. Butt, Chem. Eng. Sci. 62(11), 2843 (2007).

${ }^{6}$ S. J. Park, B. C. Petzold, M. B. Goodman, and B. L. Pruitt, Rev. Sci. Instrum. 82(4), 043703 (2011)

${ }^{7}$ N. G. Demas and A. A. Polycarpou, Tribol. Trans. 49, 291 (2006).

${ }^{8}$ E. E. Nunez, K. Polychronopoulou, and A. A. Polycarpou, Wear 270, 46 (2010).

${ }^{9}$ F. J. Giessibl, C. Gerber, and G. Binnig, J. Vac. Sci. Technol. B 9(2), 984 (1991).

${ }^{10}$ C. J. Pang and M. W. Bai, Appl. Surf. Sci. 253(2), 488 (2006).

${ }^{11}$ M. Heuberger, J. Vanicek, and M. Zach, Rev. Sci. Instrum. 72(9), 3556 (2001).

${ }^{12}$ Handbook of chemistry and physics, 55th ed. (CRC, Cleveland, Ohio, 1974).

${ }^{13}$ H. K. Christenson, J. Phys.: Condens. Matter 13(11), R95 (2001).

${ }^{14}$ M. Heuberger, M. Zaech, and N. D. Spencer, Science 292, 905 (2001).

${ }^{15}$ T. E. Balmer, H. K. Christenson, N. D. Spencer, and M. Heuberger, Langmuir 24(4), 1566 (2008)

${ }^{16}$ A. M. Stewart and H. K. Christenson, Meas. Sci. Technol. 1, 1301 (1990).

${ }^{17}$ M. Heuberger, Rev. Sci. Instrum. 72(3), 1700 (2001).

${ }^{18} \mathrm{~J}$. N. Israelachvili, Intermolecular and surface forces, 2nd ed. (Academic, London, 1985).

${ }^{19}$ A. Michels and J. Hamers, Physica 4(10), 995 (1937).

${ }^{20} \mathrm{M}$. Born and E. Wolf, Principles of Optics, 7th ed. (Cambridge University Press, Cambridge, 1999).

${ }^{21}$ C. Hertlein, L. Helden, A. Gambassi, S. Dietrich, and C. Bechinger, Nature (London) 451(10), 172 (2008).

${ }^{22}$ E. J. Wanless and H. K. Christenson, J. Chem. Phys. 101(5), 4260 (1993).

${ }^{23}$ F. Mugele and M. Salmeron, Phys. Rev. Lett. 84(25), 5796 (2000).

${ }^{24}$ T. Becker and F. Mugele, Phys. Rev. Lett. 91(16), 166104 (2003). 